\title{
REVIEW
}

\section{Emergency department overcrowding in the United States: an emerging threat to patient safety and public health}

\author{
S Trzeciak, E P Rivers
}

Emerg Med J 2003;20:402-405

Numerous reports have questioned the ability of United States emergency departments to handle the increasing demand for emergency services. Emergency department (ED) overcrowding is widespread in US cities and has reportedly reached crisis proportions. The purpose of this review is to describe how ED overcrowding threatens patient safety and public health, and to explore the complex causes and potential solutions for the overcrowding crisis. A review of the literature from 1990 to 2002 identified by a search of the Medline database was performed. Additional sources were selected from the references of the articles identified. There were four key findings. (1) The ED is a vital component of America's health care "safety net".

(2) Overcrowding in ED treatment areas threatens public health by compromising patient safety and jeopardising the reliability of the entire US emergency care system. (3) Although the causes of ED overcrowding are complex, the main cause is inadequate inpatient capacity for a patient population with an increasing severity of illness. (4) Potential solutions for ED overcrowding will require multidisciplinary system-wide support.

See end of article for authors' affiliations

Correspondence to: Dr S Trzeciak, Department of Emergency Medicine and the Section of Critical Care Medicine, Robert Wood Johnson Medical School at Camden

University of Medicine and

Dentistry of New Jersey

Cooper Hospital/University

Medical Center, Cooper

Health System, One

Cooper Plaza, 363

Dorrance, Camden,

NJ 08103, USA;

trzeciak-stephen@

cooperhealth.edu

Accepted for publication 29 June 2003
$\mathrm{N}$ umerous reports have questioned the ability of United States emergency departments to handle an increasing demand for emergency services. ${ }^{1-7}$ Emergency department (ED) overcrowding is widespread in US cities and has reportedly reached crisis proportions. ${ }^{2-9}$ The purpose of this review is to describe how ED overcrowding threatens patient safety and public health, and to explore the complex causes and potential solutions for the overcrowding crisis.

\section{METHODS}

We performed a review of relevant articles from 1990-2002 identified by a Medline search combining the MeSH heading "emergency service, hospital" with the following keywords: crowding, overcrowding, bed occupancy, health services accessibility, hospital restructuring, and patient safety. The search produced a total of 177 potential articles that were reviewed for pertinence. Of these, we selected 32 articles for this review. Additional sources were selected from the references of the articles identified. The sources included original research as well as data from peer reviewed commentaries and consensus conference proceedings.

\section{RESULTS}

America's health care "safety net" and the role of the ED

In addition to caring for acutely ill or injured patients, EDs help ensure that basic health care is available to anyone, regardless of ability to pay. ${ }^{10}$ EDs care for underserved populations who have no other options for medical care because of numerous socioeconomic barriers. For indigent patients, the uninsured, and the homeless, ${ }^{10-14}$ the ED serves as a "provider of last resort". ${ }^{15}$

In 2000, the Institute of Medicine (IOM) studied the US health care "safety net" that serves uninsured or otherwise vulnerable populations. ${ }^{16}$ The ED meets the IOM definition of safety net provider because EDs care for patients "by explicitly adopted mission, regardless of their ability to pay", and vulnerable populations comprise a "substantial share" of the ED patient mix. ${ }^{17}$

EDs, however, differ from other safety net providers in two important ways. Firstly, EDs offer access to comprehensive services within the hospital (including subspecialty care) 24 hours/day. Secondly, and most importantly, the ED is required by law to treat all patients, even when reimbursement is not guaranteed. This precedent was set by the Emergency Medical Treatment and Labor Act (EMTALA), ${ }^{18}$ a US law mandating that ED patients cannot be turned away, regardless of payer status. Because refusing patients access to the ED would be an unsafe practice, ${ }^{19}$ the Health Care Financing Administration (HCFA) now strictly enforces EMTALA, making ED services the first and only health care in the US to be guaranteed by law. ${ }^{18}$ The ED has become the only guaranteed access to health care for 44 million uninsured Americans. ${ }^{10}$ Of the 100 million patients presenting to US EDs annually, over 15 million are uninsured. ${ }^{12}$ Collectively, EDs provide more safety net care than any other safety net provider in the US. ${ }^{15}$ Because all safety net providers are extremely interdependent, ${ }^{16}$ the ED feels a much greater burden whenever other safety net providers are unable to meet the needs of the community. Essentially, the ED serves as "the safety net for the safety net." ${ }^{20}$

Therefore, the role of the ED is crucial for public health. ${ }^{10-1520}$ Any threat to the EDs ability to 
provide quality emergency care constitutes a public health crisis. Currently the greatest threat to the viability of the US emergency care system is reported to be ED overcrowding.

ED overcrowding: an emerging threat to patient safety? The potential dangers of ED overcrowding have recently garnered national attention in the United States. Numerous reports in the lay press have reported an unsafe environment in EDs because of overcrowding. Although no precise definition exists, ED overcrowding refers to an extreme excess of patients in the treatment areas, ${ }^{20}$ exceeding ED capacity and frequently necessitating medical care to be provided in ED hallways and other makeshift examination areas. Survey data in the literature have documented the broad scope of ED overcrowding in the United States. ${ }^{24-6921}$ According to a 2002 national US survey, more than $90 \%$ of large hospitals report EDs operating "at" or "over" capacity.

We are now beginning to understand the extent to which this overcrowding threatens patient safety in the ED. In survey studies, ED overcrowding has been reported to cause delays in diagnosis, delays in treatment, decreased quality of care, and poor patient outcomes. ${ }^{25}$ One report linked ED overcrowding to delays in identification and treatment of time sensitive conditions such as acute myocardial infarction, acute stroke, acute surgical emergencies, and severe sepsis. ${ }^{6}$ At least one of these cases of delayed treatment resulted in an unexpected death. ${ }^{6}$ According to the Joint Commission on Accreditation of Healthcare Organizations (JCAHO), over one half of all "sentinel event" cases of morbidity and mortality secondary to delays in treatment occur in hospital EDs, and ED overcrowding has been cited as a contributing factor in $31 \%$ of these cases. $^{22}$

ED overcrowding could potentially affect anyone who suffers unexpected severe illness or injury requiring time sensitive emergency treatment. Manifestations of ED overcrowding include (1) "boarding" of patients in the ED, (2) increased risk of medical errors, (3) ambulance diversion, (4) threat to disaster preparedness, and (5) eroding reliability of the emergency care system.

\section{(1) "Boarding" in the ED}

Many urban EDs are forced to hold patients in the ED for several hours (perhaps $>24$ hours) when inpatient beds are unavailable.9 20 23-25 "Boarding" in the ED has become a significant barrier to specialised inpatient care. ${ }^{20}$

Even critically ill patients have been reported to wait extraordinarily long periods of time in the ED until an inpatient critical care bed is available. ${ }^{29}$ For all hospitals in the US, the average waiting time for an inpatient acute or critical care bed is over three hours, but the average waiting time is 5.8 hours in hospitals with overcrowded EDs. ${ }^{2}$ Boarding in the ED could subject critically ill patients to treatment delays when time sensitive interventions are necessary. There is current evidence that in disease states such as severe sepsis, early specialised goal directed therapy within the first six hours can decrease mortality up to $16 \%{ }^{26}$ Therefore, boarding critically ill patients in a non-ICU setting could cause treatment delays at a pivotal point in the hospital course, potentially resulting in poor outcomes.

Boarding causes the ED to be filled beyond capacity with the highest acuity patients. The ED is neither designed nor equipped to provide longitudinal care, and patient safety may be compromised when there is not enough staffing in the ED to give a severely ill patient undivided attention over a long period of time. In addition, these patients may be so labour intensive that other ED patients cannot receive the necessary attention from ED staff.

(2) Increased risk of medical errors

Medical errors are frequently a byproduct of complex hospital "system" problems. ${ }^{27}$ ED overcrowding causes an environment in which quality of care may be compromised as the ED staff is pressured by the simultaneous needs of patients boarding in the ED and new patients continuously arriving. Regardless of the proficiency of the ED staff, ED overcrowding is a prime example of a system problem creating a high risk environment for medical errors and threatening patient safety. ${ }^{20}$

\section{(3) Ambulance diversion}

Ambulance diversion is another indicator of capacity constraints. $^{2}$ When EDs are overcrowded, ambulances are diverted to other institutions, and emergency medical service (EMS) crews are instructed to "bypass" an overcrowded ED even if it is the closest. Ambulance diversion has become so severe in many urban areas that EDs may be on bypass as much as $20 \%-50 \%$ of the time. ${ }^{28}{ }^{29}$ When all hospitals in a region are simultaneously on bypass, all ambulance diversions are overridden. In this scenario, nothing can be done to relieve overcrowded EDs. ${ }^{34} 29$

Timely emergency care is frequently predicated on rapid ambulance transport, and diverting to an alternative hospital may endanger patients by delaying treatment. The inability to find an open ED will also delay an EMS crew in returning to duty and responding to other emergency calls. ${ }^{24}{ }^{29}$ In effect, ambulance diversion endangers anyone who could potentially depend on EMS prompt response time. ${ }^{28-31}$

(4) A threat to disaster preparedness

The terrorist attacks of September 112001 have brought greater focus on US disaster preparedness. A key ingredient in the response to potential terrorism or bioterrorism disasters is the readiness of US EDs. Overcrowded EDs would be ill equipped to handle mass casualty victims in a disaster scenario.

(5) Eroding reliability of the United States emergency care system

As ED utilisation continues to rise, ${ }^{12}{ }^{32}$ there is great concern that US EDs will not be able to meet the growing demand for emergency services. In many regions, leaders in emergency medicine have "lost confidence in the emergency health care infrastructure" because "the current resources supporting emergency care are inadequate to meet the needs of all patients at all times." ${ }^{\prime 33}$ ED capacity has been stretched to such an extent that the quality of emergency care is reportedly eroding, and the reliability of the entire emergency care system in the US has been called into question..$^{1-5} 72331$

\section{Causes of ED overcrowding}

History

Despite managed care initiatives to limit ED visits, US ED utilisation rose by more than 14\% from 1992 to 1999, increasing to over 100 million annual patient visits. ${ }^{34}$ In the past, ED overcrowding was attributed to inappropriate use of the ED by a large volume of non-urgent patients. ${ }^{23}$ However, there has never been a consensus definition of "non-urgent", and opinions about the appropriateness of an ED visit have been widely divergent. High subsequent hospitalisation rates have been reported for patients who were initially denied ED care, ${ }^{19}$ and trying to prospectively determine the appropriateness of an ED visit has been considered a risky practice.

In addition to concerns over patient safety, the impetus to keep patients away from the ED has virtually disappeared for two reasons. Firstly, HCFA now strictly enforces EMTALA, which mandates that EDs at least provide a screening examination for all patients. ${ }^{18}$ Secondly, and most importantly, non-urgent visits are no longer believed to be the main cause of overcrowding. ${ }^{4023}$ To understand this last concept, overcrowding of the triage area (that is, waiting room) must be differentiated from overcrowding of the patient treatment areas. Non-urgent visits cause extremely crowded waiting 
rooms but reportedly do not cause crowding in the ED treatment areas, because the highest acuity patients are always brought into the treatment areas first. ${ }^{20}$ In fact, the total number of ED visits has been reported to poorly correlate with ED overcrowding. ${ }^{35}$ The notion that non-urgent patients are the main cause of the ED overcrowding crisis has now been abandoned. ${ }^{42023}$ We now realise that the true causes of ED overcrowding are much more complex, ${ }^{50}$ and include (1) inadeqate inpatient capacity, (2) higher severity of illness, and (3) hospital system restructuring.

\section{(1) Inadequate inpatient capacity}

According to the American Hospital Association, the number of inpatient hospital beds decreased 39\% in the US between 1981-1999. ${ }^{36}$ As a direct result of cost-containment initiatives, hospitals have eliminated inpatient beds in order to maintain a high census (that is, a completely "full house" at all times). ${ }^{35}$ Unfortunately, the impact on ED throughput and patient safety factors has gone unrecognised. ${ }^{37}$ Hospitals become ill equipped to handle fluctuations in demand when the average hospital census remains at or above $90 \%$ of capacity, ${ }^{38}$ resulting in an inpatient bed shortage and ED boarding. ${ }^{3}$ The greatest impediment to ED patient outflow has been the lack of inpatient hospital beds for acutely ill patients. ${ }^{24}$ Reduced inpatient capacity is reported to be the main cause of overcrowding in ED treatment areas. ${ }^{23} 202324$

Understaffing of inpatient wards with fewer nurses is another cost-containment strategy that limits inpatient capacity. ${ }^{17}$ Deliberate understaffing exacerbates the current US nursing shortage. This results in fewer staffed inpatient beds to accept patients from an overcrowded ED. ${ }^{7}$ While eliminating hospital beds and reducing staffing may make sense from a financial perspective, these cost-containment strategies fail to take into account the human toll and put patients at risk for treatment delays and bad outcomes. ${ }^{357}$

\section{(2) Higher severity of illness}

An aging population and an increasing prevalence of high complexity medical problems has increased the severity of illness among ED patients, ${ }^{3}$ and this has become an important determinant of ED overcrowding. ${ }^{8}$ For example, the number of critically ill patients presenting to California EDs from 1990 to 1999 increased by 59\%. ${ }^{8}$ This sharp rise in critical patients, coupled with a markedly decreased inpatient capacity, forces EDs to act as "pseudo-ICUs". ${ }^{3}$ In a recent report commissioned by the American Hospital Association, the most common reason for going on ambulance "bypass" was an insufficient supply of available critical care beds for critically ill ED patients. ${ }^{2}$

\section{(3) Hospital system restructuring}

Hospital system restructuring further contributes to ED overcrowding. The abrupt elimination of EDs by hospital mergers and/or closures increases the burden on neighbouring EDs. ${ }^{35}$ Nationwide from 1988 to 1996, the number of EDs fell by $9 \% .{ }^{10}$ In California, a $12.3 \%$ drop in the number of EDs from 1990 to 1999 increased the number of visits per ED by $27 \% .{ }^{8}$ The elimination of EDs has occurred at a time when the number of ED visits is higher than ever before, ${ }^{10}{ }^{39}$ increasing the annual patient visits per ED as much as $45 \%$ in some regions. $^{7}$

\section{Causes of ED overcrowding: an international perspective} ED overcrowding is not limited to the United States, and inpatient capacity has been cited as the most important factor in ED overcrowding in other countries as well. ${ }^{40} \mathrm{ED}$ overcrowding has been linked to inadequate inpatient bed availability in the United Kingdom ${ }^{38}{ }^{41}$ and Australia, ${ }^{42}$ and has been recognised as an "international symptom of health care system failure". ${ }^{43}$ Potential solutions for ED overcrowding may affect health care delivery to emergency department patients internationally.

\section{Potential solutions}

There is no easy answer for ED overcrowding. Leaders in emergency medicine have acknowledged that overcrowding will probably remain until the dangers of diminished inpatient capacity are fully recognised. ${ }^{3}$ There are, however, several measures that could help to alleviate the strain on EDs:

\section{(1) Safeguard the ED "safety net"}

We must fully recognise the unique role of the ED in the health care safety. Any threat to the integrity of the emergency care system constitutes a public health crisis. Special measures to strengthen ED infrastructure must be taken, including the allocation of state and federal resources to advance patient safety research.

\section{(2) Observation units}

One temporising measure to help alleviate the gridlock of patients awaiting admission in the ED is to provide an alternative place for them to go. ED short stay observation units have been shown to relieve ED overcrowding by giving the ED a way to control patient outflow to some extent. ${ }^{24} 4$ This is advantageous because solutions for patient outflow from the ED are expected to have the biggest impact on overcrowding. ${ }^{60}{ }^{24}$ An ED managed observation unit has been shown to markedly decrease waiting times and cut the mean monthly hours of ambulance diversion by $40 \% .{ }^{44}$ Observation units are essential in order to help EDs make room for incoming patients.

\section{(3) Early warning systems}

Local public health departments and law enforcement agencies need information systems that can integrate regional ED and hospital capacity data, detecting the early stages of ED overcrowding. When regional ED capacity is in danger of being exceeded, a civil emergency designation could trigger health department contingency plans to expand acute care capacity. Early warning systems have proved to be effective public health measures in alleviating overcrowding. ${ }^{24}$ Early warning systems may also serve as a key component of disaster preparedness. The State of New Jersey has developed the first statewide emergency radio communication system (New Jersey Hospital Communications Network) to manage acute care bed capacity in the event of a terrorist attack or other disaster scenario. ${ }^{45}$

\section{(4) Strategic planning}

The Joint Commission on Accreditation of Healthcare Organizations (JCAHO) has identified the importance of strategic planning in alleviating ED overcrowding. They recommend planning ahead for patients affected by ED overcrowding, in a similar way to planning ahead for elective surgery or elective hospital admissions. The recommendations include (1) planning for delivery of care to patients who must be placed in temporary bed locations, (2) coordination with long term health facilities and home health agencies to expedite hospital discharges, and (3) incorporating ED overcrowding initiatives into system-wide performance improvement goals. ${ }^{46}$ The strategic planning initiative is intended to help hospitals anticipate and prepare for ED overcrowding, rather than react to ED overcrowding after it has occurred.

\section{(5) Multidisciplinary approach}

Because the main causes of ED overcrowding seem to originate outside the ED, the only way to truly alleviate ED overcrowding is to focus our attention on system-wide reform. Based on 10 years of experience with ED overcrowding in Rochester, New York, Schneider et al reported that targeting internal ED efficiency had very little effect on overcrowding. ED overcrowding proved to be a complex problem deeply rooted in "issues of inpatient capacity, inadequacy of alternatives for hospitalization, and hospital resource shortages." ${ }^{24}$ 
Overcrowding was only alleviated after hospital administration and the local department of health realised that system reform was necessary. Hospital administration created a short-stay observation unit and a "transition team" to expedite the care of inpatients being boarded in the ED. The local department of health developed effective early warning systems and contingency plans. These interventions had a great impact on ED overcrowding and ambulance diversion. ${ }^{24}$ To alleviate overcrowding and better ensure the safety of ED patients, communities struggling with ED overcrowding must tackle the crisis with a similar multidisciplinary system-wide approach.

\section{CONCLUSIONS}

The ED is a vital component of the US health care safety net. ED overcrowding threatens public health by compromising patient safety and jeopardising the reliability of the entire US emergency care system. ED overcrowding is a symptom of health care system failure on multiple levels. The main cause of ED overcrowding is a failure to ensure adequate inpatient capacity for an ED population with an increasing severity of illness. Alleviating the overcrowding crisis will require a multidisciplinary system-wide approach.

\section{Authors' affiliations}

S Trzeciak, Department of Emergency Medicine and the Section of Critical Care Medicine, Robert Wood Johnson Medical School at Camden, University of Medicine and Dentistry of New Jersey, Cooper Health System, Camden, USA

E P Rivers, Departments of Emergency Medicine and Surgery, Henry Ford Hospital, Case Western Reserve University, Detroit, USA

Funding: none.

Conflicts of interest: none declared.

\section{REFERENCES}

1 Richardson LD, Hwang U. America's health care safety net: intact or unraveling? Acad Emerg Med 2001:8:1056-63.

2 Lewin Group (for the American Hospital Association). Emergency department overload: a growing crisis. The results of the American Hospital Association Survey of Emergency Department (ED) and Hospital Capacity. Falls Church, VA: American Hospital Association, 2002.

3 Derlet RW. Overcrowding in emergency departments: increased demand and decreased capacity. Ann Emerg Med 2002;39:430-2.

4 Derlet RW, Richards JR, Kravitz R. Frequent overcrowding in US emergency departments. Acad Emerg Med 2001;8:151-5.

5 Derlet RW, Richards JR. Overcrowding in the nation's emergency departments: complex causes and disturbing effects. Ann Emerg Med 2000;35:63-8

6 Derlet RW, Richards JR. Emergency department overcrowding in Florida, New York, and Texas. South Med J 2002;95:846-9.

7 Taylor TB. Threats to the health care safety net. Acad Emerg Med 2001;8:1080-7

8 Lambe S, Washington DL, Fink A, et al. Trends in the use and capacity of California's emergency departments, 1990-1999. Ann Emerg Med 2002;39:389-96.

9 Andrulis DP, Kellermann A, Hintz EA, et al. Emergency departments and crowding in United States teaching hospitals. Ann Emerg Med 1991;20:980-6.

10 Fields WE. Defending America's safety net. Report of the American College of Emergency Physicians 1998-99 safety net task force. Dallas, TX: American College of Emergency Physicians, 1999.

11 US Department of Health and Human Services. Office of the Inspector General. Use of emergency rooms by Medicaid recipients. Washington, DC: US Department of Health and Human Services, 1992

12 McCaig LF. National hospital ambulatory medical care survey: 1998 emergency department summary. Hyattsville, MD: National Center for Health Statistics, 2000.

13 Gordon JA. Emergency care as safety net. Health Aff (Millwood) 2000; 19:277.

14 Gordon JA. The hospital emergency department as a social welfare institution. Ann Emerg Med 1999;33:321-5.

15 Asplin BR. Tying a knot in the unraveling health care safety net. Acad Emerg Med 2001;8:1075-9.
16 Lewin ME, Altman SH (for the Institute of Medicine). Committee on the Changing Market Managed Care and the Future Viability of Safety Net Providers. America's health care safety net: intact but endangered. Washington, DC: National Academy Press, 2000:xviii, 281.

17 Altman SH. Statement from the chair. Committee on the changing market, managed care, and the future viability of safety net providers, 2000. http://www.iom.edu (accessed 1 Oct 2002).

18 The Emergency Medical Treatment and Active Labor Act, as established under the Consolidated Omnibus Budget Reconciliation Act (COBRA) of 1985 (42 USC 1395 dd). 1194. Federal Register, 59:32086-127.

19 Lowe RA, Bindman AB, Ulrich SK, et al. Refusing care to emergency department of patients: evaluation of published triage guidelines. Ann Emerg Med 1994;23:286-93.

20 Gordon JA, Billings J, Asplin BR, et al. Safety net research in emergency medicine: proceedings of the Academic Emergency Medicine Consensus Conference on "The Unraveling Safety Net". Acad Emerg Med 2001;8:1024-9.

21 Richards JR, Navarro ML, Derlet RW. Survey of directors of emergency departments in California on overcrowding. West J Med 2000;172:385-8.

22 Joint Commission on Accreditation of Healthcare Organizations (JCAHO). Sentinel event alert, June 17, 2002. http://www.jcaho.org/ about+us/news+letters/sentinel+event+alert/sea_26.html (accessed 21 Sep 2002).

23 Kellermann AL. Deja vu. Ann Emerg Med 2000;35:83-5.

24 Schneider S, Zwemer F, Doniger A, et al. New York: a decade of emergency department overcrowding. Acad Emerg Med 2001;8:1044-50.

25 Lynn SG, Kellermann AL. Critical decision-making: managing the emergency department in an overcrowded hospital. Ann Emerg Med $1991 ; 20: 287-92$

26 Rivers E, Nguyen B, Havstad S, et al. Early goal-directed therapy in the treatment of severe sepsis and septic shock. N Engl J Med 2001;345: 1368-77.

27 McNutt RA, Abrams R, Arons DC. Patient safety efforts should focus on medical errors. JAMA 2002;287:1997-2001.

28 Litvak $\mathbf{E}$, Long $M C$, Cooper $A B$, et al. Emergency department diversion: causes and solutions. Acad Emerg Med 2001;8:1 108-10.

29 Taylor TB. Emergency services crisis of 2000-the Arizona experience. Acad Emerg Med 2001;8: 1107-8.

30 Redelmeier DA, Blair PJ, Collins WE. No place to unload: a preliminary analysis of the prevalence, risk factors, and consequences of ambulance diversion. Ann Emerg Med 1994;23:43-7.

31 Brewster L, Rudell L, Lesser C. Emergency room diversions: a symptom of hospitals under stress. Issue brief: findings form the HSC. Washington, DC: Center for Studying Health System Change, 2001

32 McCaig LF, Stussman BJ. National Hospital Ambulatory Medical Care Survey: 1996 emergency department summary. Adv Data 1997;293:1-20.

33 Taylor TB. Position statement on the critical state of emergency care in Arizona. Arizona emergency care crisis meeting summary. Phoenix, AZ Governor's administrative offices conference, 2000.

34 Burt CW, McCaig LF. Trends in hospital emergency department utilization: United States, 1992-99. Vital Health Stat 2001;13:1-34.

35 Schull MJ, Szalai JP, Schwartz B, et al. Emergency department overcrowding following systematic hospital restructuring: trends at twenty hospitals over ten years. Acad Emerg Med 2001;8:1037-43.

36 American Hospital Association. Hospital statistics 1999. http://www.hospitalconnect.com/healthforum/hfstats/downloads.html (accessed Jul 2002)

37 Edwards N, Harrison A. Planning hospitals with limited evidence: a research and policy problem. BM 1999;319:1361-3.

38 Bagust A, Place M, Posnett JW. Dynamics of bed use in accommodating emergency admissions: stochastic simulation model. BM 1999;319:155-8.

39 American College of Emergency Physicians. EM statistical profile. http://www.acep.org/1,381,0.html (accessed Oct 2002).

40 Fatovich DM. Recent developments: emergency medicine. BM 2002;324:958-62.

41 Department of Health (UK). Reforming emergency care-practical steps, 2001 . http://www.doh.gov.uk/emergencycare/reform.htm (accessed May 2003)

42 Acute Health Division. Emergency demand management. Melbourne: Victorian Government Department of Human Services, 2001.

43 Graff L. Overcrowding in the ED: an international symptom of health care system failure. Am J Emerg Med 1999;17:208-9.

44 Kelen GD, Scheulen JJ, Hill PM. Effect of an emergency department (ED) managed acute care unit on ED overcrowding and emergency medical services diversion. Acad Emerg Med 2001 :8:1095-100.

45 State of New Jersey. Terrorism and public health emergency preparedness and response plan, October, 2002. http:// www.state.nj.us/health/er/erplan.pdf (accessed 1 Apr 2003)

46 Joint Commission on Accreditation of Healthcare Organizations. Emergency department overcrowding field review. http:// www.jcaho.org/accredited+organizations/hospitals/standards/ field+reviews/ed_fr_ltr.ht m (accessed May 2003). 